\title{
Atypical McArdle's disease with asymmetric weakness and Atrophy
}

\author{
Ratna Bhavaraju-Sanka ${ }^{1 *}$, James Howard. Jr² and Nizar Chahin ${ }^{2}$ \\ ${ }^{1}$ Department of Neurology University of Texas Health Science center San Antonio \\ ${ }^{2}$ Department of Neurology The university of North Carolina Chapel Hill
}

Received: November 09, 2013; Accepted: November 09, 2013; Published: May 15, 2014

*Corresponding author: Ratna Bhavaraju-Sanka, MD Assistant Professor of Neurology University of Texas Health Science Center San Antonio, 8300 Floyd Curl Drive, San Antonio 78229, USA, E-mail: bhavarajusan@uthscsa.edu

\begin{abstract}
McArdle's disease is an autosomal recessive disorder of glycogen metabolism caused by deficiency of myophosphorylase. It typically presents in infancy or adolescence. Adult onset cases have been reported primarily in the second decade but some late-onset cases have also been reported. The cause of fixed weakness in a metabolic myopathy like McArdle's is unclear, but repeated episodes of myoglobinuria may lead to failure of the regenerative processes resulting in atrophy and weakness. Early diagnosis could potentially prevent development of significant atrophy or fixed weakness by making the patient aware of risks of exertion and promote life style changes. We report a case of 55 year old male presenting with progressive asymmetrical weakness and atrophy. Muscle biopsy showed absent phosphorylase staining and genetic testing showed a homozygous mutation for R49X (p.Arg49Ter; c.148 C>T).
\end{abstract}

\section{Introduction}

McArdle's disease is an autosomal recessive disorder of glycogen metabolism, which occurs due to deficiency of myophosphorylase, an enzyme required for conversion of glycogen to glucose-1-phosphate in the muscle [1]. It is also known as Glycogen Storage Disease Type V or Glycogenosis Type V. This syndrome typically presents in the first two decades of life with exercise intolerance, muscle pain, cramps, rhabdomyolysis and myoglobinuria. Patients notice stiffness after exercise and a second-wind phenomenon during exercise [2]. It can present in infancy with progressive weakness, hypotonia, respiratory distress and early death. In the adult onset form, the findings are atypical with symmetric progressive limb-girdle weakness and delayed onset wasting [3]. Heart and liver have a different isomer of the phosphorylase, and are thus not affected. An ischemic exercise test can be used to screen for this condition. In a diseased person, there is absence of increase in serum lactate with an increase in ammonia production.

Histopathology of the muscle shows vacuoles filled with glycogen, [4] located predominantly in the subsarcolemmal region with absence of phosphorylase staining. The gene encoding for myophosphorylase has been localized to chromosome 11q13. The R49X nonsense mutation in exon 1 is the most common mutation in Northern Europe and North America, but 55 other distinct mutations have been identified all over the world. There has been no genotype-phenotype correlation that has been identified [5].

We report adult onset McArdle's disease in a 55-year-old male with atypical clinical features of asymmetrical onset and early atrophy. One other similar case has been reported in an 83year old male [6].

\section{Case History}

A 55-year-old right-handed male presented to our neuromuscular clinic for evaluation of progressive weakness. He retrospectively reported mild difficulties since childhood with early fatigue during physical exercise classes and inability to keep up with his peers. He noted myalgias with activity and second wind phenomenon. Few episodes of myoglobinuria could be elicited on further history

especially after strenuous activity. He denied any exercise induced cramps or contractures. He reported no previous evaluations for this problem. A neurological evaluation was sought due to development of persistent weakness and atrophy in the last 5 years. His medical history was significant for coronary artery disease with stent placement, elevated liver enzymes of unclear etiology, hypertension, hyperlipidemia and gout.

Family history was positive for a brother diagnosed of Poliomyelitis at the age of two. There was no history of neuromuscular disorders but he reports many family members with "big calves".

\section{Physical examination}

Pertinent findings on his exam include asymmetrical atrophy of his deltoids, infraspinatus, supraspinatus and quadriceps, (Figure 1) He had winging of the scapula on the right (Figure 2) and calf hypertrophy (Figure 3) His strength examination was as mentioned in Table 1. 
His reflexes were symmetric and 1+ except for absent right knee jerk. His cranial nerve, sensory and cerebellar examinations were normal.

\section{Laboratory evaluation}

There was a history of elevated CK ranging from 421-5778 U/L (70-185). He had electrodiagnostic studies performed which showed myopathic changes proximally and distally with membrane irritability and myotonic discharges. A limb girdle dystrophy panel did not reveal any pathogenic mutations

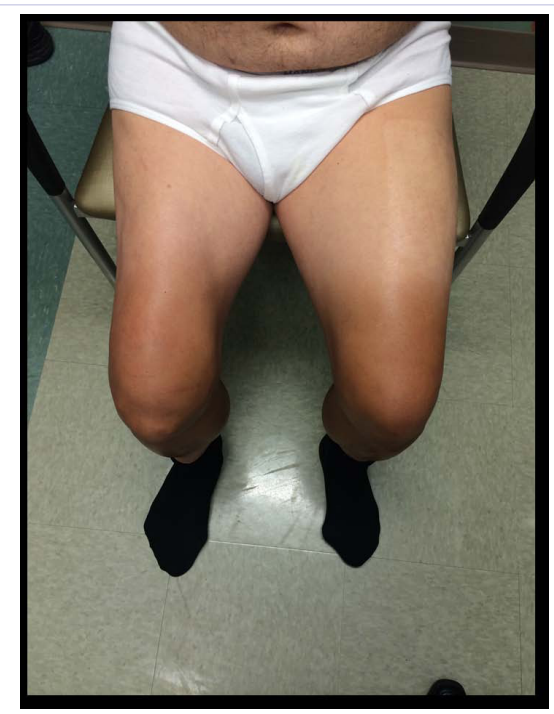

Figure 1: Atrophy of the right quadriceps muscle.

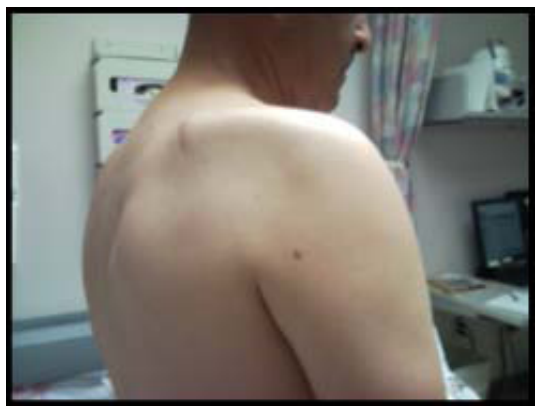

Figure 2: Periscapular atrophy with winging

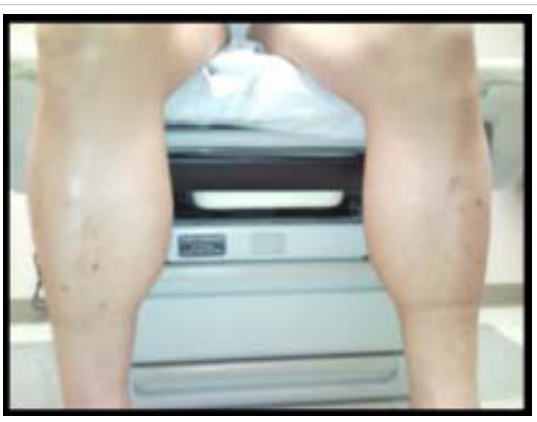

Figure 3: Calf hypertrophy.
Table 1: MRC Grading.

\begin{tabular}{|c|c|c|}
\hline Muscle & Right & Left \\
\hline Deltiod & $3-$ & 4 \\
\hline Infraspinatus & 3 & 4 \\
\hline Biceps & 5 & 5 \\
\hline Triceps & 5 & 5 \\
\hline Wrist extensors $\backslash$ flexors & 5 & 5 \\
\hline Intrinsic of hand & 5 & 5 \\
\hline Iliopsoas & $3+$ & 4 \\
\hline Quadriceps & 2 & 3 \\
\hline Hamsrtings & 5 & 5 \\
\hline Hip abductors $\backslash$ adductors & 5 & 5 \\
\hline Ankle & 5 & 5 \\
\hline Neck flexion & 4 & 5 \\
\hline Neck extension & 4 & 5 \\
\hline
\end{tabular}

in fukutin related protein, calpian 3, lamin A/c, caveolin 3, sarcoglycan and dysferlin genes. As the diagnosis was unclear muscle biopsy was performed.

\section{Histochemistry}

Left Biceps muscle biopsy was performed which showed vacuolar myopathy with subsarcolemmal accumulation of glycogen on PAS staining (Figures 4-6). There was variation is fiber size atrophy, endomysial fibrosis and rare regenerating and necrotic fibers. Phosphorylase staining in the muscle was absent. Electron microscopy showed increased subsarcolemmal glycogen along with focal increase in glycogen between the myofibrils. Biochemical analysis showed no activity of the myophosphorylase enzyme. Genetic testing showed a homozygous mutation for R49X (p.Arg49Ter; c.148 C>T).

\section{Discussion}

McArdle's disease typically presents in infancy or adolescence. Adult onset cases have been reported mostly in the second decade of life but some late onset cases have been reported [79]. Nine cases with onset after 4 th decade have been reported in literature. One case reported asymmetrical wasting at late stages of disease [10]. Five of the nine cases had symmetrical fixed weakness with one case presenting with asymmetrical weakness [11]. The cause of fixed weakness in a

metabolic myopathy like McArdle's is unclear, but repeated episodes of myoglobinuria may lead to failure of the regenerative processes resulting in atrophy and weakness. Regeneration of muscle fibers has been reported to occur only in muscle diseases with necrosis [12]. Skeletal muscle fibers usually have good capacity to regenerate due to the presence of satellite cells or myogenic precursor cells. It is predicted that these satellite cells have a limited capability of 60 mitotic cycles, after which they become senescent and lose their regenerative capability. This may be the reason for muscle atrophy and fixed weakness in later stages of McArdle's disease with necrosis from repeated exertional injury to the muscle. This does not however explain 


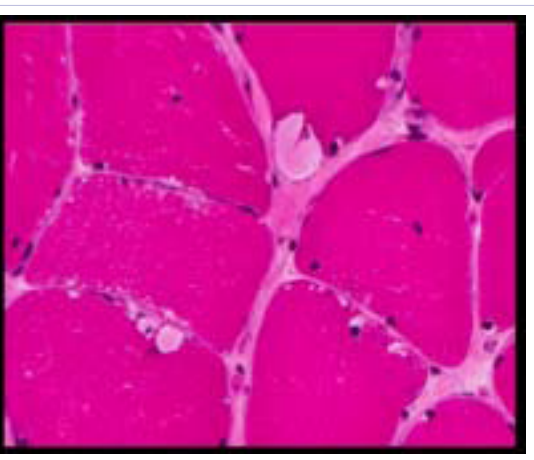

Figure 4: H\&E with subsarcolemmal vacuoles.
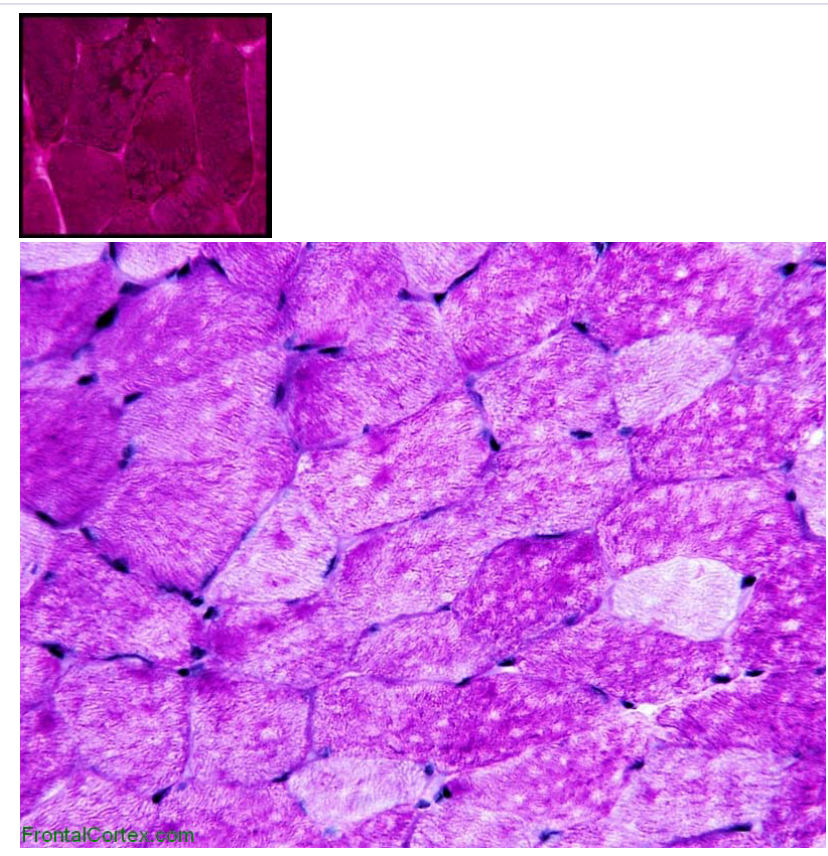

Figure 5: PAS staining showing increased glycogen with control slide on the right.

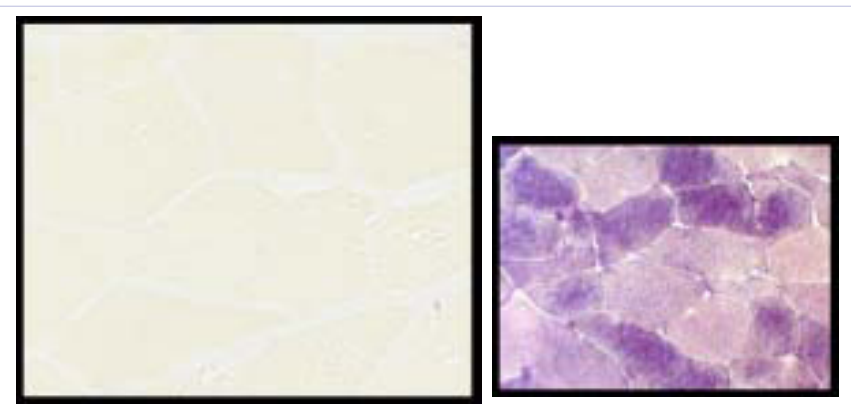

Figure 6: Absent phosphorylase staining with control on the right.

asymmetrical weakness and atrophy as seen in our case. The other case reported with similar features had compound heterozygous mutation in R49X allele. Our case had a homozygous mutation in the same gene. This strengthens the genotype- phenotype variability in this disease. It has been proposed that in patients diagnosed later in life, there is more chance of atrophy and fixed weakness as they are less likely to refrain from strenuous exercise or activity due to lack of awareness of the disease. So early diagnosis could potentially prevent development of significant atrophy or fixed weakness by making the patient aware of risks of exertion and promote life style changes.

\section{Conclusion}

Cases of late onset McArdle's disease have been reported but asymmetrical fixed weakness is rare. The occurrence of persistent weakness in McArdle's disease may be prevented by early diagnosis and life style changes to avoid recurrent rhabdomyolysis, but further prospective natural history studies are needed to support this hypothesis.

\section{References}

1. Lucia A, Nogales-Gadea G, Perez M, et al. (2008) McArdle disease: what do neurologists need to know? Nature Clinical Practice Neurology 4(10): 568- 577.

2. Pourmand R, Sanders DB, Corwin HM (1983) Late-onset McArdle's disease with unusual electromyographic findings. Archives of Neurology 40(6): 374-377.

3. McKineel IW, Rudnicki MA (2004) Molecular mechanisms of Muscle atrophy. Cell 119(7): 907-910.

4. Felice KJ, Grunnet ML, Sima AA (1996) Selective atrophy of type 1 muscle fibers in McArdle's disease. Neurology 47(2): 581-583.

5. Martin MA, Rubio CJ, Buschbinder J, et al. (2001) Molecular heterogeneity of Myophosphorylase deficiency (McArdle's disease): A genotypephenotype correlation study. Annals of Neurology:50(5): 574-581.

6. Quinlivan R, Buckley J, James M, et al. (2010) McArdle's disease: A clinical review. Journal of Neurology Neurosurgery and Psychiatry 81:1182-1188.

7. Engel WK, Eyerman EL, Williams HE (1963) Late-onset type of skeletalmuscle phosphorylase deficiency. New England Journal of Medicine 268:135-137.

8. Felice KJ, Schneebaum AB, Jones Jr HR (1992) McArdle's disease and late-onset symptoms: case report and review of the literature. Journal of Neurology Neurosurgery Psychiatry 55(5): 407-408.

9. Nadaj-Pakleza AA, Vincitorio CM, Laforet A, et al. (2009) Permanent Weakness in McArdle Disease. Muscle Nerve: 40(3): 350-357.

10. Hewlett RH, Gardner-Thorpe C (1978). McArdle's disease- What limit to the age of onset? South African Medicine 53(2):60-3.

11. Wolfe GI, Baker NS, Haller RG, Burns DK and Barohn RJ (2000). McArdle's disease presenting with asymmetric, late-onset arm weakness. Muscle \& Nerve 23(4):641-645.

12. Karpati G, Molnar MJ (2008) Muscle fibre regeneration in human skeletal muscle diseases. In: S Schiaffino and T Partridge (Eds.), Skeletal muscle repair and regeneration. Springer science+Business Media B.V. Dordrecht Netherlands, pp. 199-215. 